\title{
Blindness and visual impairment in a region endemic for onchocerciasis in the Central African Republic
}

\author{
E C Schwartz, R Huss, A Hopkins, B Dadjim, P Madjitoloum, C Hénault, V Klauss
}

University Eye Clinic

LMU, Munich,

Germany

E C Schwartz

V Klauss

German Technical

Co-operation, Projet

soins de santé

primaires, Région

sanitaire No 3,

Bossangoa, Central

African Republic

$R$ Huss

Programme National de Lutte contre

l'Onchocercose et la

Cécité (PNLOC),

Bossangoa, Central

African Republic

A Hopkins

Université de Bangui, Bangui, Central

African Republic

B Dadjim

P Madjitoloum

Biometric Center for Therapeutic Studies, Munich, Germany

C Hénault

Correspondence to: E C Schwartz, University Eye Clinic LMU,

Mathildenstrasse 8, D-80336

München, Germany.

Accepted for publication 12 February 1997

\begin{abstract}
Aims-A population based survey of blindness and visual impairment was conducted in the district of Bossangoa, Central African Republic.

Methods-A total of 48 communities were randomly selected, and 6086 people examined.

Results-The prevalence of blindness (visual acuity in the better eye less than $3 / 60$ ) was $2.2 \%$, and visual impairment $3.0 \%(6 / 24$ to $3 / 60$ in the better eye). The major causes of blindness were onchocerciasis $(73.1 \%)$, cataract $(16.4 \%)$, trachoma $(4.5 \%)$, and glaucoma $(2.2 \%)$.

Conclusion-Around $95.5 \%$ of all blindness could potentially have been prevented or treated. Ivermectin mass distribution is hoped to prevent $50 \%$ of all forms of visual loss in the future.

(Br F Ophthalmol 1997;81:443-447)
\end{abstract}

The Central African Republic is a large landlocked country of $622984 \mathrm{~km}^{2}$ with an estimated population of 3.2 million and an annual rate of population increase of $2.5 \%{ }^{1}$ With a gross national product of $\$ 290$ per head in 1986 the country is considered one of the poorest 20 in the world. Life expectancy is estimated to be 47 years for men and 52 years for women, and adult literacy rates are $55 \%$ for men and $26 \%$ for women. ${ }^{2}$ No study of the prevalence of blindness in the republic has yet been published.

A hospital and clinic based study by Potter ${ }^{3}$ provides the only published data on causes of blindness in the country. As in many African countries, ophthalmic services are poorly developed. In 1994, two ophthalmologists were working permanently in the Central African Republic, supported by a general practitioner performing eye surgery, and irregular visits from overseas ophthalmologists.

The estimated high prevalence of onchocerciasis especially in the north west led to the introduction of a vertical ivermectin distribution programme in the area in 1993. The population at risk for onchocerciasis infection is estimated to be 1.5 million for the whole country (unpublished data). The Central African Republic is among the 10 major target countries of the Africa Programme for Onchocerciasis Control which coordinates ivermectin mass distribution in 19 African countries.
In spring 1994, a population based survey was undertaken in the north western district of Bossangoa as part of the development of a primary health care and prevention of blindness programme in the area. Data on the prevalence and causes of blindness will facilitate a needs assessment and future monitoring of the impact of eye care services.

\section{Subjects and methods}

According to the results of rapid epidemiological assessments, the whole of the Central African Republic is endemic for onchocerciasis. Assessments are yet to be completed in the south western districts of the country. The north west, including the district of Bossangoa, shows the highest levels of endemicity of onchocerciasis, as well as being the most densely populated region of the country.

The district of Bossangoa with an estimated population of 100000 was chosen for a population based survey (Fig 1). The town of Bossangoa with its newly established eye hospital provided the base for the study. In order to obtain a close estimate of the population, the 1988 national census figures and census figures from the ivermectin distribution team were used to define the sampling frame for the study. A sample size of 6024 was calculated beforehand based on an expected prevalence of blindness of $3 \%$, a desired precision of $0.6 \%$ with a confidence level of $95 \%$, and a design effect of 2 . The design effect represents an attempt to quantify the consequences of a cluster sampling technique as opposed to a simple random sampling. The sample size calculation was performed using EPI 5 INFO software. ${ }^{4}$ A cluster random sampling procedure following the WHO cluster sampling recommendations for immunisation programmes was employed. ${ }^{5}$ The area was stratified by endemicity of onchocerciasis and by semiurban and rural communities. Stratification by endemicity was based on the results of a rapid assessment exercise carried out by the ivermectin distribution team. In this method endemicity of onchocerciasis is estimated by counting palpable subcutaneous nodules in the adult male population. It categorises the region into hyper-, meso-, and hypoendemic areas for onchocerciasis. ${ }^{6}$ According to the population distribution, clusters were chosen in areas of different endemicity. The region was further stratified into the semiurban area of Bossangoa town and the surrounding rural villages. A total of 48 clusters were randomly chosen, and a 


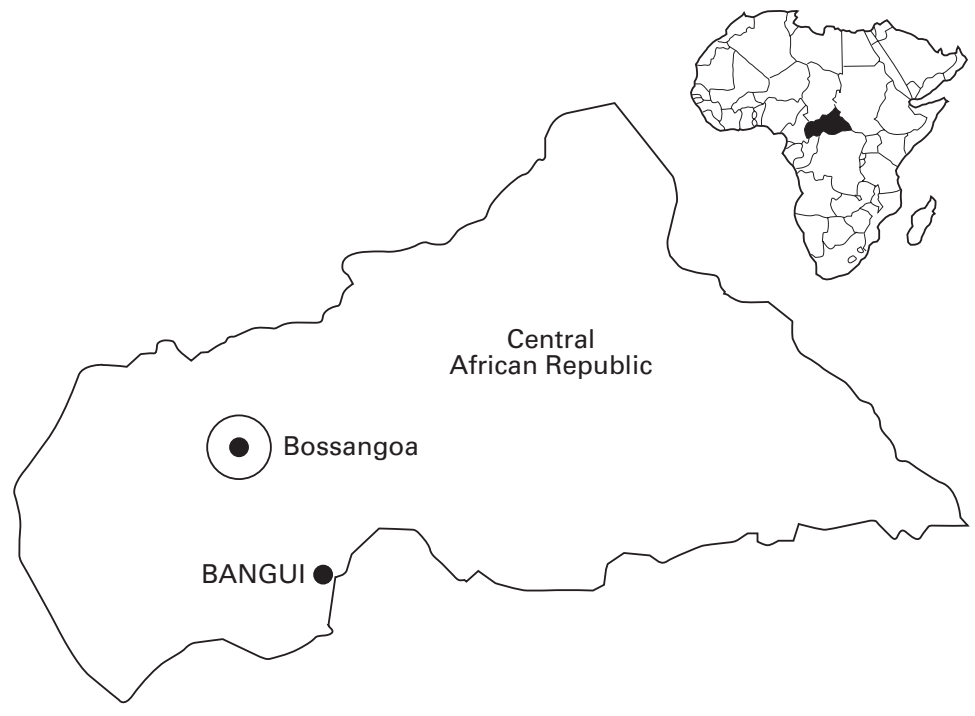

Figure 1 Map of the Central African Republic showing Bossangoa.

minimum of 150 individuals were included in each cluster. Of the 48 clusters, 30 were situated in areas hyperendemic for onchocerciasis, six in mesoendemic areas, and two in hypoendemic areas. The total sample size was 7559 individuals, of whom $6086(80.5 \%)$ were examined. Further information on the study design can be obtained from the authors.

The survey was conducted one year after the beginning of ivermectin mass distribution in the district of Bossangoa. The drug had been distributed by mobile teams visiting each community. Each person treated received a registration card stating the date and dosage of treatment. To assess treatment in our survey the information on the individual registration cards as well as recall of the person or a relative were used.

Data were collected using the Eye Examination Record (Version III) developed by the World Health Organisation Prevention of Blindness Programme, which was modified for additional information on onchocerciasis. ${ }^{7}$ The survey team consisted of two medical students trained in basic ophthalmic diagnosis, two eye auxiliaries trained in visual acuity testing, and one ophthalmologist (ECS). Demographic data were recorded including ethnicity, position within the family, occupation, and previous treatment with ivermectin. Visual acuity was measured using an illiterate E-chart, and acuities of less than $6 / 9$ were checked with a pinhole. Visual acuity in small children was estimated by testing the ability to fix centrally on a moving focal light or to recognise objects at a distance. A basic eye examination was carried out using $\times 4$ magnifying loupes, and intraocular pressure was measured by Schiøtz tonometry in all individuals above the age of 30. Assessment for trachoma in all ages and xerophthalmia (0-6 years) was carried out. In the presence of a visual acuity of less than $6 / 18$ in either eye, or any ocular pathology shown during basic eye examination, the individual was examined by an ophthalmologist. Detailed ocular examination included slit lamp biomicroscopy, direct and indirect ophthalmoscopy with dilated pupil, and gonioscopy if required. Visual field examinations and fluorescein angiography were not performed. After examination, the principal cause of impaired vision was recorded together with any additional ocular findings. All individuals were examined for the presence of palpable nodules (all ages). Any treatment required (medical or surgical) was recorded; patients were either treated immediately or referred to the eye clinic in Bossangoa. A list of cataract blind patients was established. The majority of these underwent surgery within a few months of completion of the study.

DEFINITION OF BLINDNESS AND VISUAL IMPAIRMENT

The definitions of blindness and visual impairment developed by WHO were used in the survey. ${ }^{8}$ Visual field constriction leading to blindness and visual impairment could not be considered because of logistic constraints. Unilateral blindness was defined as blindness $<3 / 60$ in one eye, the other eye being sighted $(>6 / 24)$.

\section{DIAGNOSTIC CRITERIA}

Visual impairment or blindness caused by onchocerciasis was diagnosed in the presence of typical onchocercal changes of the anterior or posterior segment which were likely to cause visual loss. ${ }^{9}$ In rare cases the diagnosis was confirmed by a skin snip taken from the iliac crest and/or shoulder of the patient. Reasons for visual loss such as sclerosing keratitis, chronic uveitis, secondary cataract, and secondary glaucoma were documented as anterior segment disease. Optic atrophy and chorioretinitis were reported as posterior segment disease. Cases of optic atrophy without clinical ophthalmic signs of onchocerciasis or any other detectable cause were recorded as optic atrophy (of unknown origin).

Glaucoma was diagnosed in the presence of typical glaucomatous cupping of the optic nerve and/or elevated intraocular pressure above $25 \mathrm{~mm} \mathrm{Hg}$.

Blindness caused by trachoma was diagnosed in the presence of entropion and trichiasis leading to corneal scarring without signs of another possible cause.

Corneal scars due to measles/xerophthalmia were diagnosed in cases where the occurrence of the ocular change dated back to early childhood with a history of severe illness. Nine cases of visual loss resulting from harmful traditional eye medicine (HTEM) were recorded where ocular symptoms had occurred after the use of traditional remedies.

\section{ANALYSIS}

Data were entered into an EPI 5 INFO database, and analysis was performed using SAS software (SAS Software Inc, Cary, NC, USA). ${ }^{5}$

\section{Results}

PREVALENCE OF BLINDNESS AND VISUAL IMPAIRMENT

Basic demographic data were obtained from a total study sample of 7559 people. Some 
Table 1 Age specific prevalence of bilateral blindness

\begin{tabular}{lll}
\hline Age (years) & Frequency of blindness & $\begin{array}{l}\text { Prevalence of blindness } \\
\text { (\%) }\end{array}$ \\
\hline $0-9$ & 0 of 2200 & 0 \\
$10-19$ & 4 of 1147 & 0.35 \\
$20-29$ & 5 of 851 & 0.59 \\
$30-39$ & 21 of 736 & 2.85 \\
$40-49$ & 28 of 486 & 5.76 \\
$50+$ & 76 of 666 & 11.41 \\
\hline
\end{tabular}

$18.6 \%$ (1408) were absent for the examination, and 65 refused an eye examination, leaving 6086 individuals examined in total. The crude prevalence of bilateral blindness was found to be 134 of $6086(2.2 \%$; 95\% confidence interval (CI) 1.83 to $2.57 \%$ ) with a sex specific distribution of $1.6 \%$ in men and $0.6 \%$ in women. Men were found to be 2.4 times more likely to be bilaterally blind than women $(\mathrm{RR}=$ $2.4 ; 95 \%$ CI 1.67 to $3.52 \%$ ).

In the villages visited, blindness prevalence rates ranged from 0 to $17 \%$. Table 1 summarises the age specific prevalence of bilateral blindness. Prevalence rates show an increase from 0 of 2200 individuals aged 0 to 9 years to 76 of $666(11.4 \%)$ in individuals above the age of 50 . For individuals in the age group defined as childhood by WHO (0-14 years), a prevalence of blindness of approximately $0.07 \%$ (2 of 2866) was found.

Visual impairment was found in 180 of 6086 individuals $(3.0 \% ; 95 \%$ CI 2.53 to $3.38 \%)$ with a distribution of $1.5 \%$ in men and $1.5 \%$ in women. Prevalence rates ranged from 2 of $2200(0.1 \%)$ in individuals aged $0-9$ years to 140 of $666(21.0 \%)$ in those above 50 years of

Table 2 Causes of visual loss in communities of the district of Bossangoa, Central African Republic

\begin{tabular}{llll}
\hline Cause & $\begin{array}{l}\text { Bilateral blindness } \\
(\text { No }(\%))\end{array}$ & $\begin{array}{l}\text { Visual impairment } \\
(\text { No }(\%))\end{array}$ & $\begin{array}{l}\text { Unilateral blindness } \\
(\text { No }(\%))\end{array}$ \\
\hline Onchocerciasis & $98(73.1)$ & $72(40.0)$ & $49(38.9)$ \\
Cataract & $22(16.4)$ & $97(53.9)$ & $36(28.6)$ \\
Trachoma & $6(4.5)$ & $3(1.7)$ & 0 \\
Glaucoma & $3(2.2)$ & 0 & $6(4.8)$ \\
Optic atrophy & $2(1.5)$ & $2(1.1)$ & $2(1.6)$ \\
HTEM & $1(0.7)$ & $4(2.2)$ & $4(3.2)$ \\
Trauma & 0 & 0 & $23(18.2)$ \\
Measles/ & & $1(0.6)$ & $4(3.2)$ \\
$\quad$ xerophthalmia & $1(0.7)$ & & \\
Micro/ & & 0 & $1(0.8)$ \\
$\quad$ anophthalmos & $1(0.7)$ & $1(0.6)$ & $1(0.8)$ \\
Refractive error & 0 & 0 & 126 \\
Aphakia & 0 & 180 & \\
Total & 134 & &
\end{tabular}

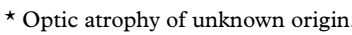

HTEM = harmful traditional eye medicine.

Table 3 Causes of blindness in areas of different endemicity of onchocerciasis

\begin{tabular}{llll}
\hline Cause & $\begin{array}{l}\text { Hyperendemic areas } \\
(\text { No }(\%))\end{array}$ & $\begin{array}{l}\text { Mesoendemic areas } \\
(\text { No }(\%))\end{array}$ & $\begin{array}{l}\text { Hypoendemic areas } \\
(\text { No }(\%))\end{array}$ \\
\hline Onchocerciasis & $92(80.7)$ & $4(28.6)$ & $2(33.3)$ \\
Cataract & $15(13 . .2)$ & $6(42.9)$ & $1(16.7)$ \\
Trachoma & $4(3.5)$ & $2(14.3)$ & 0 \\
Glaucoma & $2(1.7)$ & $1(7.1)$ & 0 \\
$\begin{array}{l}\text { Optic atrophy } \\
\text { HTEM }\end{array}$ & 0 & $1(7.1)$ & $1(16.7)$ \\
$\begin{array}{l}\text { Measles/ } \\
\quad 1(0.9)\end{array}$ & 0 & 0 \\
$\begin{array}{c}\text { Merophthalmia } \\
\quad 0\end{array}$ & 0 & 0 & $1(16.7)$ \\
anophthalmos & 0 & 0 & $1(16.7)$ \\
\hline
\end{tabular}

* Optic atrophy of unknown origin.

HTEM = harmful traditional eye medicine. age. Of those visually impaired, 50 were blind in one eye.

The prevalence of unilateral blindness was found to be approximately $2.1 \%$ (126 of 6086 , $95 \%$ CI 1.71 to $2.42 \%$ ).

CAUSES OF BLINDNESS AND VISUAL IMPAIRMENT Onchocerciasis was the single most important cause of bilateral blindness in this population, accounting for 98 of $134(73.1 \%)$ cases. The second most common cause was cataract in 22 of $134(16.4 \%)$ cases, followed by trachoma in 6 of $134(4.5 \%)$, glaucoma in 3 of $134(2.2 \%)$, and optic atrophy of unknown origin in 2 of $134(1.5 \%)$. Single cases of blindness resulting from HTEM, microphthalmos/anophthalmos, and measles/xerophthalmia accounted for the remainder.

Cataract was found to be the major cause of visual impairment in 97 of 180 (53.9\%). Onchocerciasis as the cause of visual impairment was found in 72 of 180 (40.0\%), followed by HTEM in 4 of 180 (2.2\%), trachoma in 3 of $180(1.7 \%)$, optic atrophy in 2 of $180(1.1 \%)$, refractive error in 1 of $180(0.6 \%)$, and measles/xerophthalmia in 1 of 180 individuals. The major cause of unilateral blindness was onchocerciasis in 49 of 126 cases (38.9\%), the second most common cause being cataract in 36 of 126 individuals $(28.6 \%)$. Trauma was found to be the cause of unilateral blindness in 23 of 126 cases (18.2\%), glaucoma in 6 of 126 $(4.8 \%)$, measles/xerophthalmia in 4 of 126 $(3.2 \%)$, and HTEM in 4 of $126(3.2 \%)$. Two cases of optic atrophy (1.6\%) and single cases of microphthalmos and aphakia, probably of traumatic origin, accounted for the remaining $1.6 \%$. Table 2 shows an overview of the distribution of causes of visual loss in the communities studied.

PREVALENCE AND CAUSES OF BLINDNESS IN AREAS OF DIFFERENT ENDEMICITY FOR ONCHOCERCIASIS Table 3 shows the distribution of causes of blindness in the areas of different endemicity for onchocerciasis. In the hyperendemic area, which accounted for 5954 of $7542(78.9 \%)$ of the total study population, the prevalence of blindness was found to be 114 of 4559 (2.5\%; $95 \%$ CI 2.05 to $2.95 \%$ ). A total of 1249 $(16.6 \%)$ of the study population were living in mesoendemic areas. The prevalence of blindness was here found to be 14 of $1196(1.2 \%$; $95 \%$ CI 0.59 to $1.81 \%)$. The prevalence of blindness in areas hypoendemic for onchocerciasis was 6 of $331(1.8 \% ; 95 \%$ CI 0.37 to $3.23 \%$ ). Hypoendemic areas accounted for 339 of $7542(4.5 \%)$ of the total study population.

Onchocerciasis was the single most important cause of blindness in the hyperendemic area accounting for 92 of $114(80.7 \%)$. Other causes of blindness in this area were cataract in 15 of $114(13.2 \%)$, trachoma in 4 of 114 (3.5\%), glaucoma in 2 of $114(1.7 \%)$, and HTEM in 1 of $114(0.9 \%)$. The most common cause of blindness in mesoendemic areas was cataract, accounting for 6 of $14(42.9 \%)$. The second most common cause in these areas was onchocerciasis in 4 of $14(28.6 \%)$, followed by 
trachoma in 2 of $14(14.3 \%)$, and single cases of glaucoma and optic atrophy of unknown origin $(7.1 \%)$.

The six cases of blindness found in areas hypoendemic for onchocerciasis were due to onchocerciasis in two of six $(33.3 \%)$, and single cases $(16.7 \%)$ of cataract, optic atrophy of unknown origin, microphthalmos, and measles/xerophthalmia.

COVERAGE WITH IVERMECTIN

The coverage with ivermectin in the communities visited was estimated to be $41.8 \%$ (3160 of 7559 ). A total of 3061 of the study population $(40.5 \%)$ had taken the drug once and 99 $(1.3 \%)$ more than once.

\section{Discussion}

The prevalence of blindness in the area endemic for onchocerciasis was found to be $2.2 \%$. Several factors led to a probable underestimation of the true burden of blindness and visual impairment in the community. First and foremost, no visual field testing was performed in our study. The expected high amount of visual loss due to visual field constriction in endemic onchocercal communities was therefore left undetected. A survey of mesoendemic onchocercal communities in Kaduna State, Nigeria, found an additional $0.6 \%$ of blindness resulting from field constriction. The prevalence of blindness in people aged 5 years and over in that study was $2.7 \%$ by acuity criteria alone. ${ }^{10}$ Secondly, our figures do not take into account blind children staying at the blind school of Bossangoa. Finally, 1408 people were absent for the examination, most of them staying out in the fields for several days. When only those absent individuals that were reported to be blind by their relatives were considered, the prevalence of blindness among the absentees was 27 of $1408(1.9 \%)$. We therefore do not agree with the assumption of others that absentees are likely to be sighted. From our observations, blind people actively participate as far as is possible in work in the fields, and some have been trained to do so by the rehabilitation centre in Bossangoa.

Individuals living in communities hyperendemic for onchocerciasis account for 5968 of $7559(78.9 \%)$ of the whole study sample. It is therefore not surprising that the majority of blindness in the study population is due to onchocerciasis. The prevalence of bilateral blindness from onchocerciasis alone was 98 of $6086(1.6 \%)$. Onchocerciasis was associated with all forms of visual loss in 219 of 6086 $(3.6 \%)$ individuals in the study population. About $50 \%$ (219 of 440) of all visual loss was of onchocercal origin, and could potentially have been prevented by ivermectin treatment. The excess of bilateral blindness in men as a result of onchocercal infection observed in this study has previously been reported by several authors. ${ }^{10-12}$ Reasons for this could be hormonal, genetic, immunological, or differences of exposure.

Cataract is responsible for bilateral blindness in 22 of $6086(0.36 \%)$ of the study population.
This finding is comparable with data from surveys in other African countries. A countrywide population based study in the Gambia showed a prevalence of blindness of $0.7 \%$, approximately $0.3 \%$ of which was due to cataract and uncorrected aphakia. ${ }^{13}$ In Kaduna State, Nigeria, $0.26 \%$ of the population examined were blind because of cataract. ${ }^{10}$

If this is extrapolated to the total population of the Central African Republic, approximately 11500 people are bilaterally blind from cataract, and some 51200 visually impaired. However, onchocerciasis leads to a reduced life expectancy in heavily infected areas. ${ }^{12}$ It may be assumed that the proportion of elderly people and therefore the prevalence of cataract in areas with a low endemicity of onchocerciasis is even higher. In view of the numbers of cataract blind in the country, facilities and personnel for cataract surgery are urgently needed.

Blinding trachoma was found in six cases, all of which were middle aged and elderly women with corneal scars or phthisis bulbi caused by trichiasis. Only two cases of active inflammatory trachoma were found in children.

Owing to the lack of visual field assessment, the prevalence of glaucoma in the population is likely to be underestimated by our study. Clinical observations of ophthalmologists in the country report frequent cases of visual loss resulting from primary open angle glaucoma in patients. Potter ${ }^{3}$ found glaucoma to be the cause in $12.7 \%$ of bilaterally blind attending eye clinics.

As much as $95.5 \%$ of all bilateral blindness in the study population is either curable or could have been prevented. Blindness resulting from infectious diseases, onchocerciasis, cataract, and HTEM is potentially preventable through health education. Future challenges for those concerned with eye health in the Central African Republic are the provision of basic eye services as part of a primary health care system, the training of cataract surgeons, and the implementation of a sustainable system for ivermectin distribution at the community level.

This work was completed with the cooperation, material, and financial assistance of the German Technical Co-operation, the Committee for the Prevention of Blindness in Germany, and Christoffel Blindenmission. It was supported by the Universities of Munich, Germany and Bangui, Central African Republic.We gratefully acknowledge the untiring work of the entire survey team, and the assistance of Dr P Buch on the team, and the assistance of Dr P Buchmann and Dr B AzeufakFoster and Dr J Whitworth for their inspiring advice during the study.

1 WHO. World Health Statistics Annual 1994. Geneva: WHO, 1995: c3-c8

2 United Nations. Demographic Yearbook 1993. New York: United Nations, 1995: 264

3 Potter AR. Causes of blindness and visual handicap in the Central African Republic. Br f Ophthalmol 1991;75:326-8.

4 Burton AH, Dean JA, Dean AG. Software for data management and analysis in epidemiology. World Health Forum 1990;11:75-7.

5 WHO. The Expanded Programme on Immunization Coverage Survey. Geneva: WHO, 1991: WHO/EPI/MLM/91.10.

6 WHO. Onchocerciasis and its control. Report of a WHO Expert Committee on onchocerciasis control. Geneva: WHO, 1995, WHO Technical Report Series 852.

7 WHO. World Health Organization programme for the prevention of blindness. Eye examination record, version III. Unpubtion of blindness. Eye examination record, version III. Unpub-
lished mimeographed document. Geneva: WHO, 1988: WHO/PBL/88.1. 
8 WHO. WHO methods of assessment of avoidable blindness. Geneva: WHO, 1980: WHO offset publication no 54.

WHO. WHO Expert Committee on Onchocerciasis Third report. Geneva: WHO, 1987: WHO Technical Report Series, no 752

10 Abiose A, Murdoch I, Babalola O, Cousens S, Liman I, Onyema J, et al. Distribution and aetiology of blindness and visual impairment in mesoendemic onchocercal communities, Kaduna State, Nigeria. Br f Ophthalmol 1994;78:8-13.
11 Budden FH. The epidemiology of onchocerciasis in northern Nigeria. Trans R Soc Trop Med Hyg 1956;50:366-78.

12 Kirkwood B, Smith P, Marshall T, Prost A. Relationships between mortality, visual acuity and microfilarial load in the area of the Onchocerciasis Control Programme. Trans $R$ Soc Trop Med Hyg 1983;77:862-8.

13 Faal H, Minassian D, Sowa S, Foster A. National survey of blindness and low vision in The Gambia: Results. $\mathrm{Br} \mathcal{F}$ Ophthalmol 1989;73:82-7 